American Journal of Applied Sciences 5 (8): 1079-1082, 2008

ISSN 1546-9239

(C) 2008 Science Publications

\title{
Novel Approach to Determine the Effects of MS Environment using the Portable GPS Receiver with Built-in Antenna
}

\author{
Wan Azlan Wan Zainal Abidin, Kiyotaka Fujisaki and Mitsuo Tateiba \\ Department of Computer Science and Communication Engineering, \\ Kyushu University, 744 Motooka, Nishi-ku, Fukuoka, 819-0395, Japan
}

\begin{abstract}
Communication impairment on the Mobile Satellite (MS) path for non-geostationary satellite is mainly due to effect of multipath and shadowing. In order to study the signal quality received by the MS user, a receiver system that has similar characteristics to the actual receiver used by the MS user should be used. This study presents a novel approach to determine the MS signal quality by using a portable, handheld and commercially available Global Positioning System (GPS) receiver with built-in small-size antenna that imitates the actual receiver used by the MS users. It offers a low-cost and simple to manage method of studying the MS environment. Propagation data for the open space environment are measured and used as reference to determine the MS signal quality for the tree shadowed and building shadowed environments. Comparisons are made with the results obtained using different type of receiver system to show the feasibility of this type of receiver.
\end{abstract}

Key words: Mobile satellite, attenuation, GPS receiver, multipath, shadow environment

\section{INTRODUCTION}

Two main sources of signal degradation in the MS system are shadowing effect and multipath fading. Many investigations have been performed at different frequency bands ${ }^{[1,2]}$ and have addressed issues such as the effect of shadowing and multipath on the MS signal.

Recently, there have been many studies carried out using the GPS to study the MS channel. However, the GPS system used comprised of high performance GPS receiver with highly sensitive externally connected GPS antenna ${ }^{[3,4,5,6]}$ specifically designed for the purpose of doing experimental work. Therefore, the aim is to imitate the actual MS receiver's performance by using a commercially available, portable, handheld type of GPS receiver with built-in small-size antenna which has been designed mainly for the purpose of navigation and positioning, similar to the characteristics of the actual MS receiver.

Furthermore, investigations done using the existing geo-satellite system or airborne platform ${ }^{[1,2]}$ are complex and costly which prevent more experimental works to be carried out for different region in the world. With this method, the cost and complexity will be greatly reduced.

Since this type of GPS receivers have not been designed for the purpose of experimental work, it is important to perform some calibration processes before the effect of multipath and shadowing can be studied.

The MS channel characteristics will be evaluated in terms of the SNR signal fading time-series with respect to the open space reference data and also the Cumulative Distribution Function (CDF) of fading signal at any given probability.

\section{RESEARCH METHODOLOGY}

In our experimental work, we used a commercially available, portable handheld Garmin eTrex GPS receiver that can track up to 12 satellites simultaneously to obtain propagation data such as signal-to-noise-ratio (SNR), elevation angle and azimuth. The propagation data are automatically recorded on a portable computer for later analysis based on the National Marine Electronic Association (NMEA) 0183 protocol. Data from each satellite is identified from their respective pseudorandom number (PRN); PRN3 indicates signal from satellite with PRN number of 3.

The data acquisition system is shown in Fig. 1. The receiver was placed on a flat platform with the display panel and the built-in patch antenna facing the sky. The radiation pattern of the patch antenna used in this GPS receiver allows perfect signal reception from boresight with the response attenuated as elevation angle

Corresponding Author: Wan Azlan Wan Zainal Abidin, Department of Computer Science and Communication Engineering, Kyushu University, 744 Motooka, Nishi-ku, Fukuoka, Japan Tel: +8192-8023631 Fax: +81928023600 


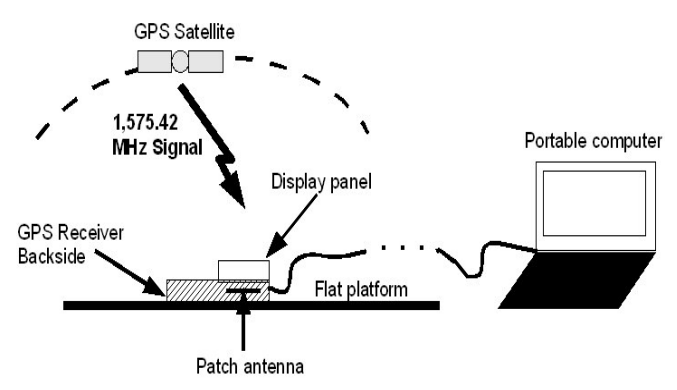

Fig. 1: Data acquisition system

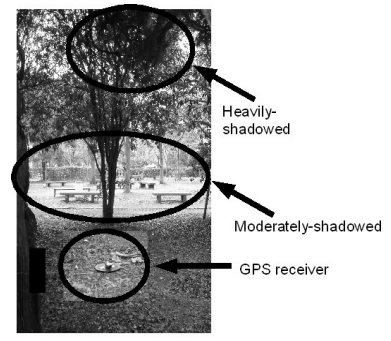

(a)

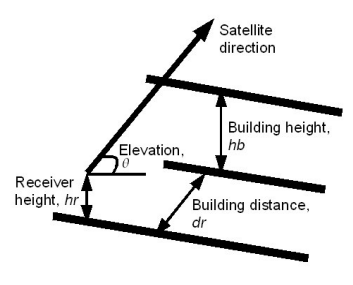

(b)
Fig. 2: Tree-shadowed and building-shadowed environment

decreases. This receiver will have lost of signal lock when the received SNR is less than $30 \mathrm{dBHz}$. Whenever this condition occurs, the received SNR will be given as $0 \mathrm{dBHz}$. The highest measured SNR is 54 dBHz.

For the purpose of this study, data measurements have been carried out at Hakozaki Campus in Kyushu University, Japan under the fine weather condition. The open space measured data are used as reference to the measured data for tree-shadowed and buildingshadowed condition.

The experimental set up used to study the effect of tree shadowing and that of building are shown in Fig. 2a and $b$ respectively. For these set up, the receiver was also placed flat facing the sky and measurements were carried out under the fine weather condition.

\section{RESULTS AND DISCUSSION}

Figure 3 shows the fading characteristics for the open space measurements of seven different satellites that orbit the sky of Fukuoka measured using this receiver. These fading characteristics have a very good agreement between each other which imply that the fading characteristics for the open space environment are the same without the presence of significant shadowing and multipath effects. At $10 \%$ probability, the fading is about $4 \mathrm{~dB}$ and this result agrees with that

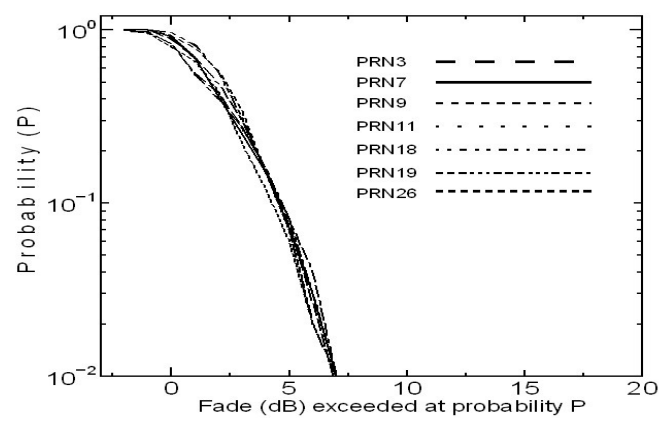

Fig. 3: The CDF of satellites that are visible across the sky of Fukuoka

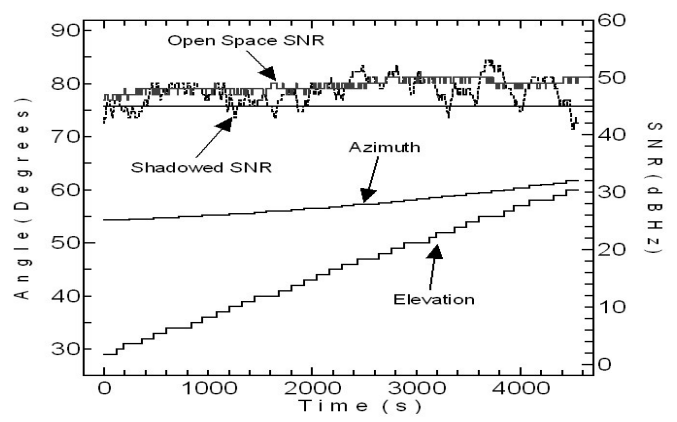

Fig. 4: The measured SNR for the moderatelyshadowed tree environment (PRN19)

obtained by $\operatorname{Lin}^{[6]}$ for outdoor scenario in which a sensor board type of GPS receiver has been used in the experimental work.

Most importantly, the fading characteristics show that the receiver used is this experimental work has a very high reliability in terms of the measured SNR values and therefore, the use of the open space result as reference is justified in order to study the effect of fading on the actual MS receiver due to obstacles such as trees and buildings.

This calibration process must be performed since this type of GPS receiver is designed mainly for navigation and positioning purpose rather than for carrying out experimental work.

Shown in Fig. 4 is the time-series of the fading signal PRN19 for the moderately-shadowed tree environment. The solid line represents the open space SNR while the dotted line is the shadowed SNR. The comparison between the open space and the moderately-shadowed SNR was made over the same 
elevation and azimuth range. The received SNR has good agreement with the open space SNR but experienced more fluctuation effect due to the presence of tree leaved and branches. The presence of some opening also allows the satellite signal to be received.

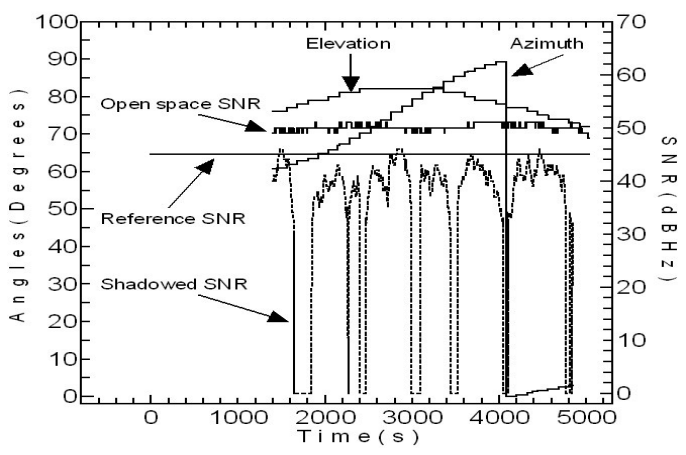

Fig. 5: The measured SNR for the heavily-shadowed tree environment (PRN3)

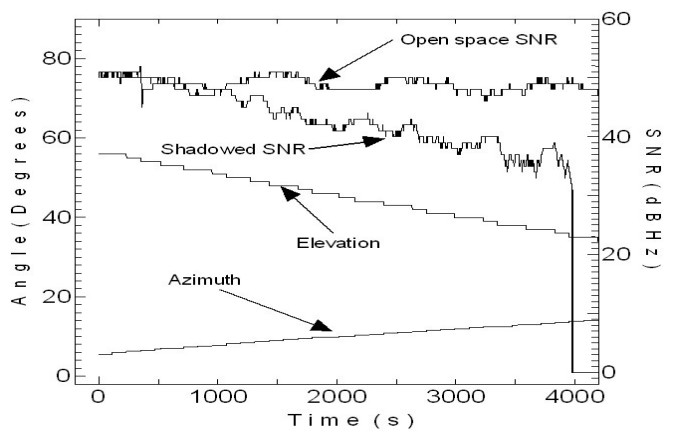

Fig. 6: The measured SNR for the building-shadowed environment (PRN26)

Figure 5 shows the measured SNR of PRN3 for the heavily-shadowed tree environment. Also in Fig. 5, the comparison between the open space and the heavilyshadowed SNR was made over the same elevation and azimuth range. The effect of heavy shadowing due to high density of tree branches and leaves greatly reduced the strength of the received signal. The lost of signal lock indicated by the $0 \mathrm{dBHz}$ received signal value could also be observed which means that the received signal quality is not acceptable for the communication purpose.

Figure 6 shows the measured SNR of PRN26 for the building-shadowed condition. By analyzing the received SNR of the satellite signal arriving from the direction where the building was present, the fading effect of the building was determined. Comparison made with the open space data over the same elevation and azimuth range shows that the building can attenuate the received signal strength. At measurement time of 4000 s for example, the total lost of signal $(0 \mathrm{dBHz})$ can be observed due to the total blockage of the incoming signal by the building.

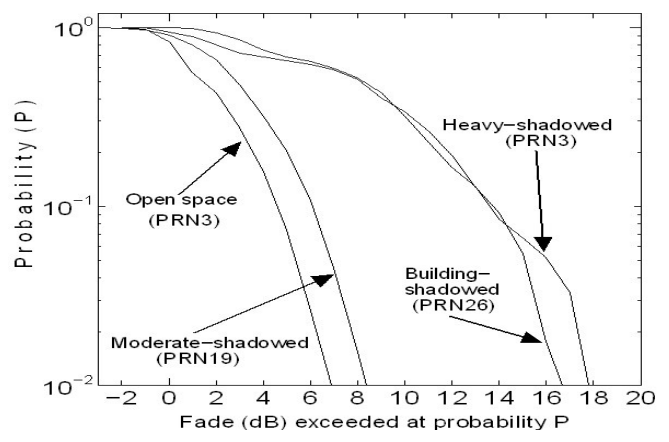

Fig. 7: Fading characteristics for different MS environments

In Fig. 7, the MS fading characteristics for different environments are shown. At $10 \%$ probability, the fading is $4 \mathrm{~dB}, 7 \mathrm{~dB}, 14 \mathrm{~dB}$ and $14 \mathrm{~dB}$ for the open space, moderately-shadowed, heavily-shadowed and the building-shadowed environments. Our result for the open space and the moderately-shadowed tree environment is comparable to that obtained by $\operatorname{Lin}^{[6]}$ in which a sensor board type of GPS receiver was used for the experimental work. Depending on the density of tree leaves and branches, the effect of the treeshadowing could be as severe as for the building environment.

\section{CONCLUSION}

The proposed experimental method of using the commercially available, portable, handheld, GPS receiver with built-in small-size antenna can closely imitate the actual MS receiver's signal performance due to their similar characteristics. By using the open space results as reference, the effect of fading was determined. Preliminary results obtained for the open space and tree-shadowed environments show good agreement with that obtained using sensor board type of GPS receiver while the effect of building can also be determined using the same experimental set up. This method allows measurements to be carried out with much reduced cost and less complicated measurement set up while providing reliable received signal quality 
that can be used to determine the effect of fading in the MS environments.

\section{ACKNOWLEDGEMENT}

This research was partly supported by the $21 \mathrm{st}$ Century COE Program 'Reconstruction of Social Infrastructure Related to Information Science and Electrical Engineering' in 2006.

\section{REFERENCES}

1. Goldhirsh, J. and W.J. Vogel, 1998. Handbook of propagation effects for vehicular and personal mobile satellite systems: Overview of experimental and modeling results, http://www.utexas.edu/research/mopro/papercopy/ handbook.pdf.

2. Smith, H., J.G. Gardiner and S.K. Barton, 1993. Measurements on satelite-mobile channel at $\mathrm{L}$ and S bands, Proc. 3rd Int. Mobile Satellite Conf., IMSC'93.
3. Kirchner, M. and M. Becker, 2005. The Use of Signal Strength Measurements for Quality Assessments of GPS Observations, Reports on Geodesy No. 2 (73), http:www.fomi.hu/cegrm/ public/Kirchner_Becker_05.pdf.

4. Hurtado, M., H. Lorente and C. Muravchik, 2001. An In-situ Method to Measure the Radiation Pattern of a GPS Receiving Antenna, IEEE Transaction Instrumentation and Measurement.

5. Hatsuda, T., Y. Yamada, J. Osada, T. Kobayashi, T. Kato and Y. Aoki, 2000. Mobile broadcast Geostationary satellite system propagation measurements using GPS signal. IEEE Antenna and Prop. Soc. International Symposium.

6. Lin, H.P. and M.J.F. Tseng, 2002. Analyzing GPS signals to investigate path diversity effects of nonstationary orbit satellite communication systems. Int. J. Satellite Communicat. 